\title{
CALIDAD DE VIDA EN PACIENTES CON MELANOMA DE COROIDES
}

\section{QUALITY OF LIFE IN PATIENTS WITH CHOROIDAL MELANOMA}

\author{
BLANCO-RIVERA C ${ }^{1}$, CAPEÁNS-TOMÉ C ${ }^{2}$, OTERO-CEPEDA XL ${ }^{3}$
}

\section{RESUMEN}

Objetivo: Determinar qué tratamiento, la braquiterapia con $\mathrm{I}^{125}$ o la enucleación, aporta una mayor calidad de vida a los pacientes tratados de melanoma de coroides.

Pacientes y método: Se administró una versión modificada del cuestionario VF-14 a 65 pacientes tratados de melanoma coroideo mediante braquiterapia, enucleación o ambos. Los datos obtenidos fueron sometidos a un estudio estadístico.

Resultados: Se encontraron diferencias estadísticamente significativas entre la agudeza visual media del ojo afecto y la visión binocular, inicial y final, sufriendo ambas un importante descenso después del tratamiento. La puntuación media global del cuestionario VF sufrió también un descenso estadísticamente significativo después del tratamiento.

Conclusión: Se puede afirmar que los pacientes tratados mediante braquiterapia tuvieron una mejor percepción de su salud general que los pacientes enucleados, alcanzando mejores puntuaciones en las tareas del cuestionario VF. Por otra parte, la agudeza visual binocular y el tiempo transcurrido después del tratamiento se presentaron como factores influyentes en la calidad de vida percibida por los pacientes.

Palabras clave: Calidad de vida, melanoma de coroides, braquiterapia, enucleación, VF-14.

\section{ABSTRACT}

Purpose: To determine whether iodine 125 brachytherapy or enucleation is better for quality of life in patients treated for choroidal melanoma.

Patients and methods: Sixty-five patients with choroidal melanoma were treated with a modified version of VF-14 iodine 125 brachytherapy, enucleation or both. Results of this treatment were then statistically analyzed.

Results: Statistically significant differences were found between visual acuity in the affected eye and binocular vision (initial and final), with an important decline after the treatment. The global medial score in the VF questionnaire decreased in a statistically significant manner after the treatment.

Conclusion: We can confirm that patients treated with brachytherapy had better health-related quality of life than those who had their eye enucleated and were more likely to have higher (better) scores on the VF questionnaire items. On the other hand, the binocular vision and time after the treatment correlated with the quality of life in long-term follow-up (Arch Soc Esp Oftalmol 2008; 83: 301-306).

Key words: Quality of life, choroidal melanoma, brachytherapy, enucleation, VF-14.

\footnotetext{
Recibido: 25/5/07. Aceptado: 14/4/08.

Servicio de Oftalmología del Complexo Hospitalario Universitario de Santiago. Santiago de Compostela. La Coruña. España.

1 Doctora en Medicina. Servicio de Oftalmología. Complexo Hospitalario Universitario de Vigo (CHUVI).

2 Doctora en Medicina.

3 Profesor titular del Departamento de Bioestadística. Universidad de Santiago de Compostela.

Comunicación presentada en el LXXX Congreso de la S.E.O. (Valencia 2004).

Correspondencia:

M. ${ }^{a}$ Carmen Blanco Rivera

C/. Salvadas, $31,2 .{ }^{\circ} \mathrm{C}$

15705 Santiago de Compostela (Coruña)

España

E-mail: mcarmenbr@wanadoo.es
} 


\section{INTRODUCCIÓN}

El melanoma de la úvea es el tumor intraocular maligno primario más frecuente en el adulto, con una incidencia anual que oscila entre seis y siete casos por millón de habitantes/año en Estados Unidos (1). A pesar de los avances diagnósticos y terapéuticos de los últimos años, la incidencia de metástasis no se ha reducido de forma sustancial (2).

En los años 70, las teorías de Zimmerman, hicieron pensar que el tratamiento mediante enucleación favorecía la diseminación metastásica, pero hoy día los estudios más recientes, parecen demostrar que esto no es así, y ya podrían existir micrometástasis en el momento de la enucleación (3). Por otra parte, los estudios realizados por el Collaborative Ocular Melanoma Study Group (C. O. M. S.) concluyeron con una supervivencia similar entre los pacientes tratados de melanoma de coroides mediante braquiterapia con $\mathrm{I}^{125}$ y aquellos que fueron enucleados $(4,5)$.

Teniendo en cuenta estos datos del C. O. M. S., la controversia se plantea ahora respecto a cuál de estos dos tratamientos aporta una mayor calidad de vida a los pacientes tratados de melanoma de coroides. Así pues, en los últimos años han proliferado los estudios encaminados a resolver esta incógnita $(1,6,7)$ siendo el estudio más amplio el realizado por el C. O. M. S. (8-10).

Por otra parte, la calidad de vida es un concepto que cada vez está más presente en la Medicina actual. La Organización Mundial de la Salud (O.M.S.) la definió, en 1994, como «la percepción personal de un individuo de su situación en la vida, dentro del contexto cultural y de valores en que vive, y en relación con sus objetivos, expectativas, valores e intereses» (11). La calidad de vida se puede definir, también, como la valoración subjetiva del impacto de una enfermedad o de un tratamiento en el bienestar tanto físico, psíquico, como social del individuo (6). Su cuantificación es compleja y para ello el investigador dispone de una serie de instrumentos de medición o cuestionarios, que deben reunir unos requisitos y cada uno de ellos tiene, a su vez, unas características determinadas (12). En este estudio se ha seleccionado el cuestionario VF-14, al que se le realizaron una serie de modificaciones convirtiéndolo en VF-19, el cual fue ampliamente validado antes de ser utilizado (13). La decisión de utilizar el VF-14 se fundamentó, principalmente, en las características de este cuestionario, que fue diseñado para ser utilizado en pacientes con cataratas y que se ha empleado en numerosos estudios de diversas patologías oculares $(12,13)$.

\section{SUJETOS, MATERIAL Y MÉTODO}

En base a una serie de criterios de inclusión y exclusión, se realizó una selección entre los pacientes tratados de melanoma de coroides. Los pacientes debían reunir los siguientes criterios para ser introducidos en el estudio: haber recibido el tratamiento al menos 6 meses antes, que dicho tratamiento fuese braquiterapia con $\mathrm{I}^{125}$, enucleación o ambos, y que la afectación por el tumor hubiera sido monocular. Se excluyeron del estudio aquellos pacientes que presentaron melanoma con extensión a cuerpo ciliar y/o a iris, aquellos con afectación severa de la visión del otro ojo y los enfermos con edad avanzada o con problemas psicológicos que impidiesen la realización del cuestionario.

A los pacientes seleccionados, se les realizó una exploración oftalmológica completa, registrando la mejor agudeza visual en ambos ojos. Se calculó también la visión binocular mediante una media ponderada de la visión del mejor ojo (multiplicada por 0,75 ) y la del peor ojo (multiplicada por 0,25 ).

Se obtuvo autorización de los pacientes, mediante un consentimiento informado, para participar en el estudio. El cuestionario seleccionado fue el VF-14, al que se le realizaron modificaciones convirtiéndolo en VF-19, añadiéndole, además, preguntas destinadas a valorar la satisfacción de los pacientes con la asistencia recibida, su opinión sobre la información administrada respecto a su enfermedad, sobre la valoración de su experiencia personal durante el tratamiento, conocer la opinión de los pacientes sobre su estado de salud en general y la calidad de vida subjetiva percibida por los mismos (Anexo 1).

La entrevista se llevó a cabo personalmente en 50 casos, cuando el paciente acudió a la consulta y, en 15 casos fue realizada por teléfono. En todos los casos fue la misma persona la que realizó dicha entrevista (la autora de este trabajo), después de recibir un entrenamiento en el manejo del cuestionario, así como en la forma de realizar las preguntas. Finalmente, se llevó a cabo un estudio estadístico de los datos obtenidos. Se utilizaron los coeficientes de correlación de Pearson y de Spearman para evaluar la relación entre la agudeza visual (AV) y las respuestas al cuestionario VF. Se aplicó la prueba t-Student apareada para comparar los valo- 


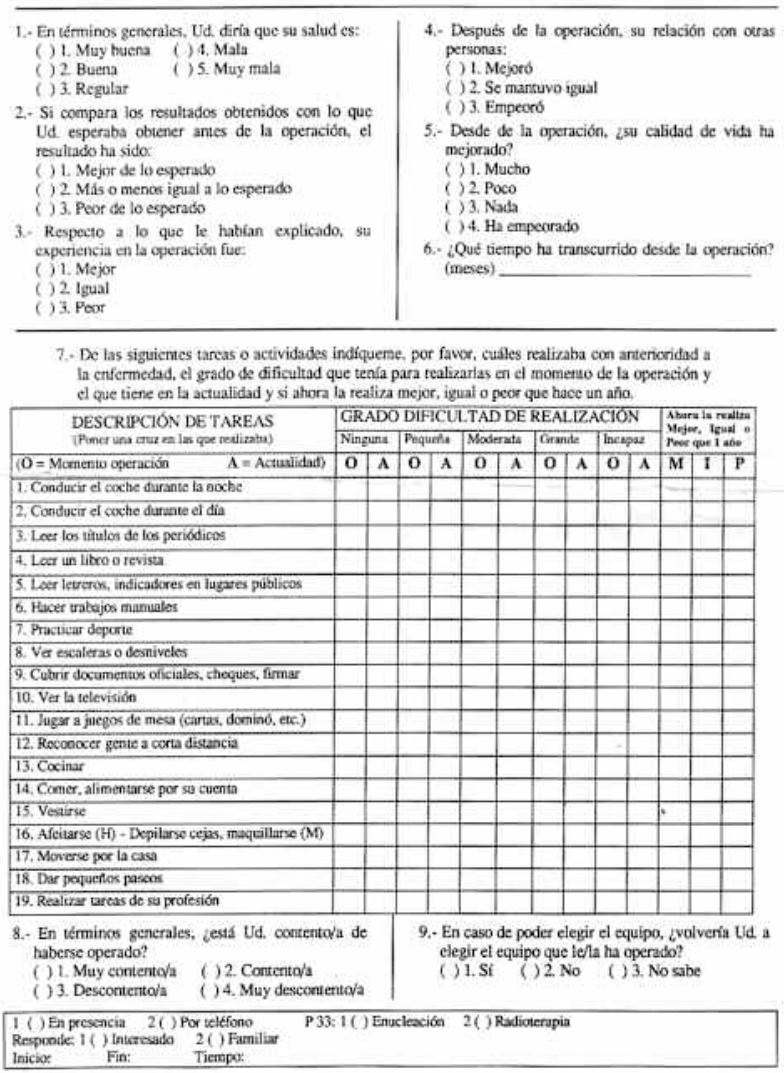

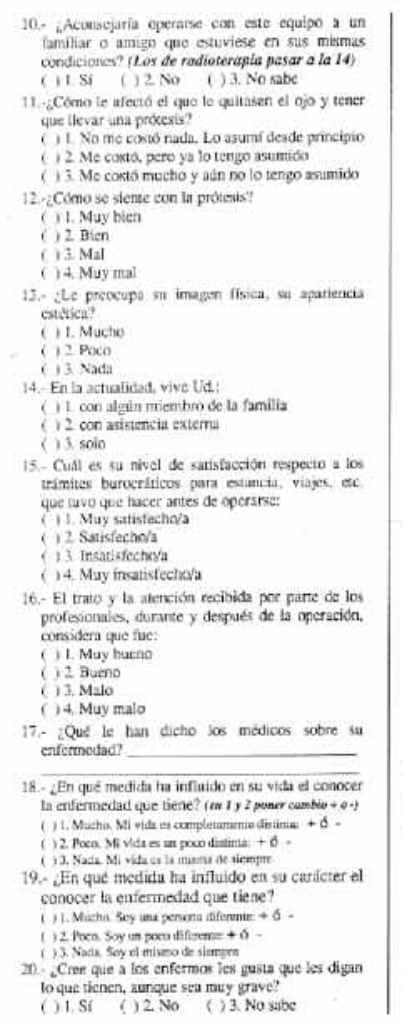

21. Inteque, por favor, si considera verdaderas $(V)$ o filsas (F) lak siguientes afimaciones

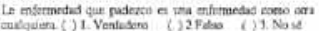

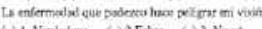

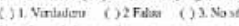

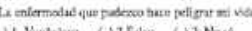

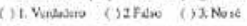
22. Corsidera que ta información çe xe le ha daco (5: 6) 1. Buena () 2. Regular () 3. Mala Comprensible () 2 Incompremsibid 1. Suficiente ( ) 2. Regular ( ) 3. Insoliciente. 23.- En general, cull as su opinita sobte el funcionemiemo del Servicio de Ontalmologia del

1. Muy buena

1. Mata

4. Muy mala

Desearianis confirmar una exric de tuos personalo parantizáncole en todo moracato el anovimato de las expuestis datio

24.- Nomibor

Howbre () 2. Mujer

Edad

Muricipio

9.- Teléforos

10. Estutios acalarios:

L. Prufosiont

DATOS TÉCNICOS

32. Otgano afecado 1,6$)$ OD $2,(\})$

3. Tipo de tratamient:

C) Eruclacicion

() 2 Radotcrapia

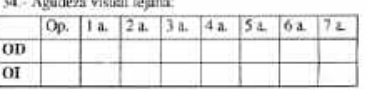

35.- Complicaciones, observaciones bal labi de ats

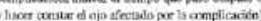

Anexo A: Modelo del cuestionario VF-19(VF- 14 modificado) utilizado en el estudio.

res de las variables antes y después del tratamiento. Y para la comparación entre tratamientos se aplicó el ANOVA de medidas repetidas. Para el análisis estadístico se empleó el programa SPSS 12.0 (SPSS for Windows, SPSS Inc., Chicago, USA).

\section{RESULTADOS}

Finalmente, se seleccionaron para el estudio 65 pacientes, 29 de los cuales fueron varones y 36 mujeres. La edad media de los entrevistados fue de 64,47 años. Respecto al tratamiento, fueron tratados con braquiterapia 45 pacientes, se enuclearon 14 enfermos y seis pacientes se trataron primero con radioterapia y posteriormente fueron enucleados. Hay que destacar que sólo el 12,3\% de los pacientes pertenecían al área sanitaria y el 69,23\% fueron remitidos desde otras comunidades. El seguimiento de los pacientes osciló entre los 6 y los 120 meses.
A los 3 años del tratamiento con radioterapia (el $100 \%$ de los pacientes radiados alcanzaron esa supervivencia frente a un $80 \%$ de los que fueron enucleados) se encontró un descenso estadísticamente significativo de la visión respecto a la situación previa al tratamiento $(\mathrm{p}=0,002)$ y la agudeza visual en un $64 \%$ de los pacientes radiados, a los 3 años, fue igual o inferior a 0,100 . La visión binocular también mostró un descenso estadísticamente significativo en el grupo de pacientes radiados $(\mathrm{p}<0,001)$, pero no existieron diferencias estadísticamente significativas entre el grupo de pacientes radiados y los que fueron enucleados $(p=0,768)$. En la figura 1 se expone la relación entre los valores iniciales y finales de la visión binocular en los tres grupos de tratamiento, mostrando un deterioro en la agudeza visual que fue estadísticamente significativo.

En cuanto al cuestionario, se presentó un descenso en la puntuación previa al tratamiento $(3,89 \mathrm{DE} 0,24)$ y posterior al mismo $(3,66 \mathrm{DE} 0,30)$, 


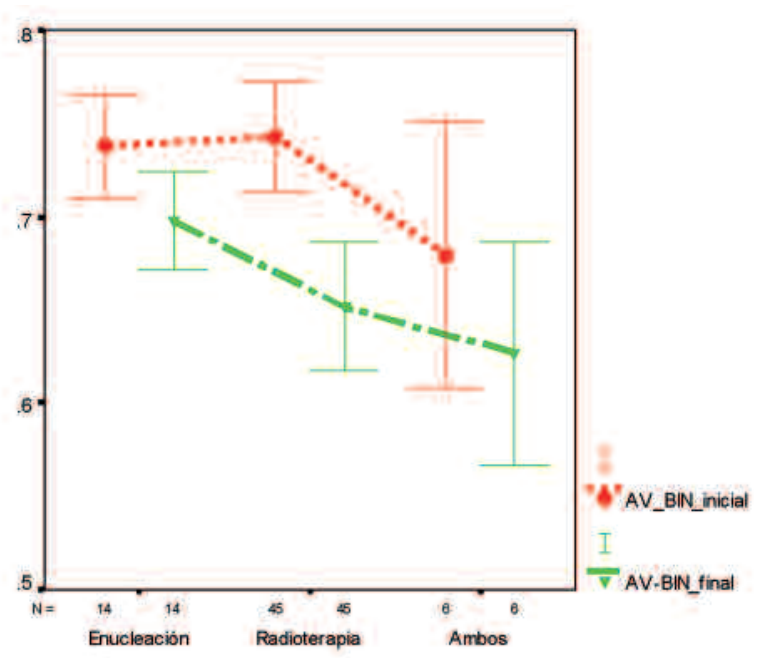

Fig. 1: Relación entre los valores iniciales y finales de las agudezas visuales binoculares en los tres grupos de tratamiento.

que resultó estadísticamente significativo $(\mathrm{p}<0,001)$. Las diferencias entre el grupo de enucleados y el de pacientes radiados fueron estadísticamente significativas a favor de los segundos $(\mathrm{p}=0,008)$. En la tabla I se muestran los valores de las puntuaciones del VF antes y después del tratamiento en cada uno de los grupos de tratamiento y su significación estadística.

La correlación entre la agudeza visual binocular y la puntuación global del VF fue mayor con el coeficiente de Spearman que con el de Pearson, tanto antes $(0,23)$ como después del tratamiento $(0,20)$. La correlación de la visión del ojo afecto y la puntuación global del VF también fue mayor con el coeficiente de Spearman, sobre todo después del tratamiento $(0,43)$.

Las tareas más afectadas fueron las de conducir, tanto por el día como por la noche, leer un libro o revista, ver escaleras o desniveles, cubrir cheques o documentos $(p<0,001)$, reconocer gente a corta distancia $(\mathrm{p}=0,017)$ y realizar tareas de su profesión ( $\mathrm{p}$ $=0,027$ ). Sólo se apreciaron diferencias estadísticamente significativas entre el grupo de enucleados y el grupo de pacientes radiados en las tareas de: leer un libro o revista, ver escaleras o desniveles, cubrir cheques o documentos y reconocer gente a corta distancia ( $p=0,015)$. Se encontraron también diferencias estadísticamente significativas $(p=0,002)$ entre la valoración del estado de salud general que tienen los pacientes radiados frente a los que fueron enucleados, siendo mejor la percepción del mismo por los radiados que por los enucleados. También se reflejó que los pacientes acababan asumiendo su enfermedad, aunque el $87,5 \%$ de los enucleados reconocieron que la enfermedad afectaba de forma negativa a su vida frente al $64 \%$ de los pacientes radiados. Sin embargo, aunque un $61,5 \%$ de los pacientes afirmaron ser conscientes de lo que implicaba su enfermedad, sólo un 33,8\% reconocieron que ésta hacía peligrar su vida. La mayoría de los pacientes reconocieron estar satisfechos con el trato recibido por los profesionales del centro durante el periodo que duró el tratamiento. En cuanto a los pacientes que fueron enucleados, estos, en su mayoría, afirmaron, haber asumido la pérdida del órgano y sentirse bien con la prótesis.

Se observó una relación entre la calidad de vida subjetiva percibida por los pacientes y la agudeza visual binocular final, de tal manera que cuanto mayor era la agudeza visual después del tratamiento, mejor era la calidad de vida percibida por los pacientes (fig. 2). También se apreció la relación existente entre la calidad de vida subjetiva percibida por los enfermos y el tiempo transcurrido desde la intervención, de tal manera que a medida que el tiempo transcurrido desde la intervención era mayor, la percepción de la calidad de vida que tenían los pacientes mejoraba (disminución del estrés, miedo, ansiedad...) (fig. 3).

\section{DISCUSIÓN}

Transcurridos 3 años después del tratamiento, los pacientes radiados sufrían una importante pérdida de agudeza visual, lo mismo que ocurría en los estu-

Tabla I. Valores de las puntuaciones del cuestionario VF antes y después del tratamiento

\begin{tabular}{lccc}
\hline Tipo de tratamiento & Puntuación inicial & Puntuación final & Significación estad. \\
\hline Enucleación & $3,81 \pm 0,40$ & $3,41 \pm 0,35$ & $\mathrm{P}=0,004$ \\
Radioterapia I- 125 & $3,91 \pm 0,18$ & $3,74 \pm 0,26$ & $\mathrm{P}<0,001$ \\
Ambos tratamientos & 4,00 & $3,64 \pm 0,20$ & $\mathrm{P}=0,009$ \\
\hline \hline
\end{tabular}




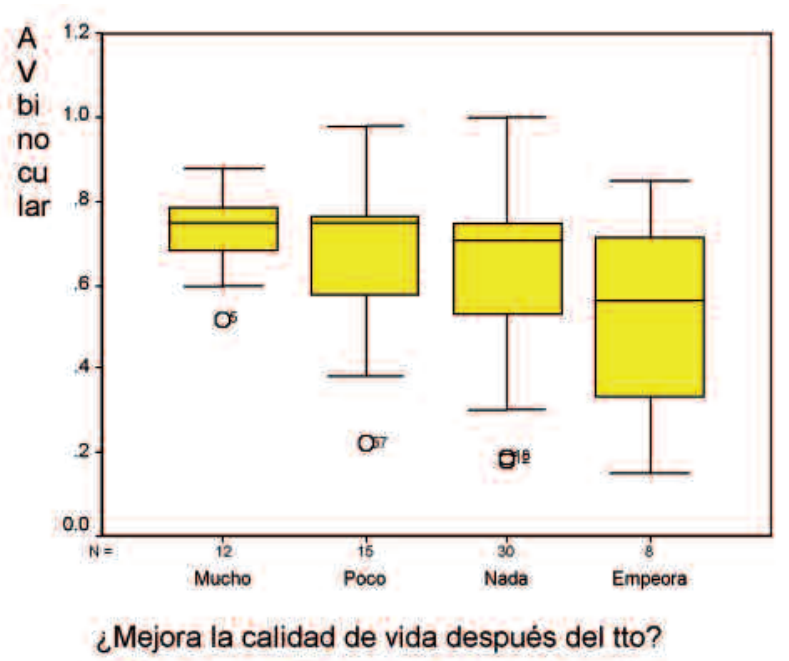

Fig. 2: Relación entre la agudeza visual binocular y la evolución de la calidad de vida subjetiva percibida por los pacientes.

dios realizados por el C.O.M.S. (14), donde el $49 \%$ de sus pacientes presentaban a los 3 años visiones inferiores a 0,100 y en los realizados por Shields et al (14) en el que a los 10 años de seguimiento, el $68 \%$ de los pacientes radiados presentaban agudezas visuales muy bajas. En cuanto a la visión binocular, no se encontraron diferencias estadísticamente significativas entre ambos grupos de tratamiento, aunque la agudeza visual binocular sí disminuyó de forma significativa después del mismo.

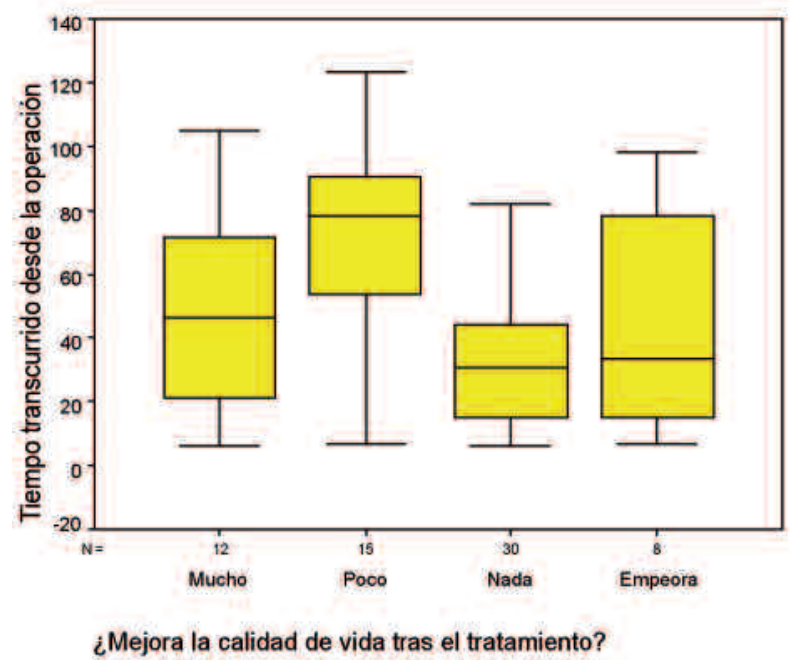

Fig. 3: Relación entre la calidad de vida percibida por los pacientes y el tiempo transcurrido desde que han recibido el tratamiento.
Queremos destacar que ningún paciente se negó a realizar el cuestionario VF, el cual demostró ser fácil de aplicar y de comprender por parte de los pacientes, al mismo tiempo que se reveló como un instrumento eficaz para valorar la visión funcional de los mismos, correlacionándose su puntuación con la agudeza visual que presentaron los enfermos. Se mostró un descenso significativo en la puntuación de las tareas que requirieron una mayor discriminación visual, como la de conducir, en la que no se encontraron diferencias entre el grupo de pacientes radiados y el de enucleados, igual que ocurrió en el estudio realizado por Cruickshanks y col. (1). En cuanto a la percepción de su estado de salud general, destacar que ésta fue más positiva por parte del grupo de pacientes radiados, de forma similar al estudio realizado por Cruickshanks y col.(1). Reimer y col. encontraron en su trabajo (6) que el nivel de stress mental se correlacionaba con el nivel de agudeza visual, siendo ésta más deficiente en el grupo de pacientes enucleados. Sin embargo, y de forma paradójica, los enfermos enucleados percibieron una mejoría más importante en su calidad de vida subjetiva que los que fueron radiados, dato que podría explicarse por el Síndrome de Damocles (miedo de una recurrencia de la enfermedad, que sería menor en estos pacientes al extirpar el órgano afectado, al contrario que los enfermos radiados que lo conservarían). Con el paso del tiempo, la mayor parte de los pacientes afirmaron aceptar su enfermedad y esto explicaría la mejoría en la percepción de su calidad de vida. Del mismo modo, los resultados finales obtenidos por el COMS (10) y posteriores a nuestro estudio, presentaron conclusiones similares a las presentadas en nuestro trabajo, ellos encontraron que sus pacientes presentaban mayor dificultad en la tarea de conducir y en la visión periférica, siendo ligeramente menor dicha dificultad en los pacientes radiados frente a los enucleados, pero, por otra parte, coinciden con nuestro trabajo en que los pacientes radiados presentaron mayores niveles de ansiedad que los enucleados, disminuyendo estos a medida que transcurre el tiempo desde la intervención y mejorando su calidad de vida subjetiva.

Por tanto, se pueden extraer varias conclusiones, que serían, por una parte, que el VF resultó ser un instrumento eficaz para valorar la calidad de vida en pacientes tratados de melanoma de coroides y que su aplicación fue sencilla, pudiendo realizarse, incluso, de forma rutinaria en la consulta. Por otro lado, los pacientes radiados presentaron menor difi- 
cultad que aquellos que fueron enucleados para realizar tareas de dificultad intermedia como ver escaleras o desniveles, leer libros o revistas, cubrir documentos oficiales y reconocer gente a corta distancia, mientras que ambos grupos de pacientes tuvieron grandes dificultades para realizar el grupo de tareas más minuciosas y prácticamente no plantearon dificultad para realizar las tareas más sencillas.

Y, además, la agudeza visual final y el tiempo de evolución de la enfermedad se revelaron como los factores que más influyeron en la percepción que los pacientes tuvieron sobre su calidad de vida.

Así pues, ante dos opciones terapéuticas que ofrecen una supervivencia similar y que, según los estudios más recientes, al igual que en nuestro trabajo, no presentan importantes diferencias en cuanto a calidad de vida se refiere, parece aconsejable valorar de forma personalizada e individual la elección de uno u otro tratamiento, teniendo en cuenta también la preferencia personal de cada paciente.

\section{BIBLIOGRAFÍA}

1. Cruickshanks KJ, Fryback DG, Nondahl DM, Robinson $N$, Keesey U, Dalton DS, et al. Treatment choice and quality of life in patients with choroidal melanoma. Arch Ophthalmol 1999; 117: 461-467.

2. Capeans C. Melanomas de la úvea. Madrid: Sociedad Española de Oftalmología; 1993.

3. Singh AD, Rennie IG, Kivela T, Seregard S, Grossniklaus H. The Zimmerman-McLean-Foster hypothesis: 25 years later. Br J Ophthalmol 2004; 88: 962-967.

4. The Collaborative Ocular Melanoma Study (COMS) randomized trial of pre-enucleation radiation of large choroidal melanoma II: initial mortality findings. COMS report no 10. Am J Ophthalmol 1998; 125: 779-796.

5. Diener-West M, Earle JD, Fine SL, Hawkins BS, Moy CS, Reynolds SM, et al. The COMS randomized trial of iodine 125 brachytherapy for choroidal melanoma, III: initial mortality findings. COMS Report No. 18. Arch Ophthalmol 2001; 119: 969-982.
6. Reimer J, Esser J, Fleiss A, Hessel A, Anastassiou G, Krausz M, et al. Quality of life in patients with malignant choroidal melanoma after radiotherapy. Graefes Arch Clin Exp Ophthalmol 2003; 241: 371-377.

7. Melia MB, Moy SC, McCaffrey L. Quality of life in patients wiht choroidal melanoma: a pilot study. Ophthalmic Epidemiol 1999; 6: 19-28.

8. Quality of life assessment in the collaborative ocular melanoma study: desing and methods. COMS-QOLS Report No. 1. COMS Quality of Life Study Group. Ophthalmic Epidemiol 1999; 6: 5-17.

9. Melia M, Moy CS, Reynolds SM, Cella D, Murray TG, Hovland KR, et al. Development and validation of disease- specific measures for choroidal melanoma: COMSQOLS report No. 2. Arch Ophthalmol 2003; 121: 10101020.

10. Melia M, Moy CS, Reynolds SM, Hayman JA, Murray TG, Hovland KR, et al. Quality of life after iodine 125 brachytherapy vs enucleation for choroidal melanoma: 5-year results from the Collaborative Ocular Study: COMS QOLS Report No 3. Arch Ophthalmol 2006; 124: 226238.

11. Casado J, González, N, Moraleda S, Orueta R, Carmona J, Gómez-Calcerrada RM. Calidad de vida relacionada con la salud en pacientes ancianos en atención primaria. Aten Primaria 2001; 28: 167-173.

12. Prieto L, Prieto L, Badía X. Cuestionarios de salud: concepto y metodología. Aten Primaria 2001; 28: 201-209.

13. Blanco-Rivera MC. Calidad de vida en pacientes tratados de melanoma de coroides. Santiago de Compostela: Universidad de Santiago de Compostela. 2005; 198 pp. Thesis doctoralis.

14. Linder M, Chang TS, Scott IU, Hay D, Chambers K, Sibley $L M$, et al. Validity of the visual function index (VF-14) in patients with retinal disease. Arch Ophthalmol 1999; 117: 1611-1616.

15. Melia BM, Abramson DH, Albert DM, Boldt HC, Earle JD, Hanson WF, et al. Collaborative Ocular Melanoma Study (COMS) randomized trial of I-125 brachytherapy for medium choroidal melanoma, I. Visual acuity after 3 years COMS report no. 16. Ophthalmology 2001; 108: 348-366.

16. Shields CL, Shields JA, Carter J, Gündüz K, Miyamoto C, Micaily $B$, et al. Plaque radiotherapy for uveal melanoma: long-term visual outcome in 1106 consecutive patients. Arch Ophthalmol 2000; 118: 1219-1228. 